\title{
Erratum to: Untersuchung der Dämpferwicklung und des natürlichen Dämpfersystems der Schenkelpol-Synchronmaschinen
}

\author{
Andrei Nicolaide
}

Published online: 14 September 2014

(C) Springer-Verlag Berlin Heidelberg 2014

\section{Erratum to: Electr Eng}

\section{DOI 10.1007/BF01574840}

Typesetting errors in some formulas were discovered. The correct versions are listed below:

$$
\begin{aligned}
& k_{d}=-\frac{2 \alpha \sin ^{2} \frac{\alpha \pi}{2}}{\gamma-1}+\frac{\sin ^{2} \frac{\alpha \pi}{2}+(\gamma-1)}{\gamma-1} c_{\alpha} ; \\
& c_{1 d}=\int_{0}^{y_{1}} \frac{\mathrm{d} y}{\delta_{y}} . \\
& K_{d}=\frac{1}{2 \sin ^{2} \frac{\alpha_{c}}{2}}\left[2+\frac{n_{c}}{4} \cdot \frac{b_{D}}{S_{d}}\right] \frac{\alpha_{c}}{\pi},
\end{aligned}
$$

\footnotetext{
A. Nicolaide $(\bowtie)$

Transilvania University of Brasov, Brasov, Romania

e-mail: andrei.nicolaide@gmail.com
} 